\title{
ASSOCIAÇÃO ENTRE A TSM DO ATLÂNTICO NORTE E A CONVECÇÃO DE VERÃO SOBRE A AMÉRICA DO SUL - SIMULAÇÕES NUMÉRICAS
}

\author{
Rosane Rodrigues Chaves
}

Recebido em 26 janeiro, 2006 / Aceito em 26 junho, 2006

Received on January 26, 2006 / Accepted on June 26, 2006

\begin{abstract}
The association between sea surface temperature (SST) over North Atlantic Ocean (Equator to $40^{\circ} \mathrm{N}$ and $70^{\circ} \mathrm{W}$ to $0^{\circ}$ ) and summer convection over South America is studied through numerical simulations with the CPTEC atmospheric general circulation model. SST anomalies over North Atlantic have influence only in the summer convection over the northernmost region of the South America, with warm SST anomalies there associated with convection over this continental region.

Keywords: North Atlantic, South America, sea surface temperature, convection, atmospheric model.

RESUMO. Neste estudo a associação entre a temperatura da superfície do mar (TSM) do 0 ceano Atlântico Norte (Equador e $40^{\circ} \mathrm{N} \mathrm{e} 70^{\circ} \mathrm{W}$ a $0^{\circ}$ ) e a convecção de verão sobre a América do Sul é examinada através de simulações numéricas com o modelo atmosférico do Centro de Previsão de Tempo e Estudos Climáticos (CPTEC). Os resultados obtidos mostram que a influência da TSM do Atlântico Norte sobre a convecção da América do Sul é significativa apenas na costa norte da América do Sul no período de dezembro-janeiro-fevereiro. A convecção sobre esta região está associada com águas quentes sobre a região do Atlântico Norte aqui considerada. Assim, a TSM sobre a área aqui considerada do Atlântico Norte tem pouca influência na previsibilidade da convecção sobre maior parte da América Sul nos meses de verão no modelo do CPTEC, corroborando os resultados de Chaves (2005a) obtidos através de análise observacional para DJF de 1979 a 2001.
\end{abstract}

Palavras-chave: Atlântico Norte, América do Sul, temperatura da superfície do mar, convecção, modelo atmosférico. 


\section{INTRODUÇÃ̃o}

Sabe-se da importância das anomalias de temperatura da superfície do mar (TSM) do Atlântico Norte para a convecção do norte do Nordeste Brasileiro (NEB; Hastenrath \& Heller, 1977; Moura \& Shukla, 1981; Nobre \& Shukla, 1996). No entanto, existem poucos trabalhos avaliando a importância das anomalias de TSM do Atlântico Norte na convecção de verão da América do Sul. Chaves (2005a) mostra através de análise observacional com os dados do Comprehensive Ocean-Atmosphere Data Set (COADS) e da reanálise do National Centre for Atmospheric Research and National Center for Atmospheric Research (NCEP/NCAR) que a influência da TSM do Atlântico Norte sobre a convecção da América Sul limita-se ao setor norte da América do Sul nos meses de dezembro-janeiro-fevereiro (DJF) para o período de 1979 a 2001. Assim, os resultados deste artigo indicam que a previsibilidade da convecção sobre maior parte da América Sul nos meses de verão tem pouca dependência das condições da TSM sobre o Atlântico Norte para o período mencionado acima e também considerando a metodologia empregada.

Alguns autores têm estudado a influência do Oceano Atlântico tropical sobre a precipitação da região Amazônica equatorial. Fu et al. (2001) através de experimentos numéricos mostram que a TSM do Atlântico tropical exerce influência na precipitação da parte leste desta região nas estações equinociais e nas estações de solstício esta influência é reduzida. Liebmann \& Marengo (2001) verificam que a TSM do Atlântico Equatorial está positivamente correlacionada com a precipitação do leste da Amazônia durante 0 outono e a primavera. Segundo estes autores a precipitação de dezembro-janeiro-fevereiro (DJF) nesta região não apresenta correlação significativa com a TSM do Atlântico equatorial. Buchmann et al. (1990) verificam através de experimentos numéricos que as secas na parte central da América do Sul no verão de 1986 e 1988 estão associadas ao aquecimento anômalo da região tropical norte e sul do Atlântico, com precipitação abaixo (acima) da média sobre o NEB quando este aquecimento encontra-se ao norte (sul) do equador.

Diversos fatores contribuem para modular a variabilidade da convecção sobre a América do Sul e áreas adjacentes, como por exemplo, a variabilidade da TSM do Oceano Pacífico tropical e do Atlântico. Desta forma, não é possível determinar isoladamente a importância das anomalias de TSM do Oceano Atlântico Norte sobre a convecção da América do Sul através de métodos observacionais. Uma forma de avaliar o efeito da TSM sobre a atmosfera de uma determinada região é através de experimentos numéricos usando modelos de circulação da atmosfera com imposição de anomalias de TSM em uma região específica. Assim, neste estudo foram conduzidos experimentos com o modelo de circulação geral da atmosfera do CPTEC para avaliar a importância da TSM do Atlântico Norte na convecção de verão da América do Norte e avaliar os resultados obtidos através de análise observacional em Chaves (2005a).

Enquanto os artigos citados acima consideram 0 Atlântico tropical, neste artigo considera-se apenas a região do Atlântico Norte entre 0 Equador e $40^{\circ} \mathrm{N}$, como em Chaves (2005a). Este procedimento é feito para avaliar separadamente a influência do setor norte do Atlântico sobre a convecção de verão da América do Sul e também como forma de eliminar o problema da correlação interhemisférica artificial que uma análise envolvendo a parte norte e sul desta Bacia pode ocasionar (Houghton \& Tourre, 1992; Dommenget \& Latif, 2000). Na seção seguinte são apresentados 0 modelo e a metodologia empregados nos experimentos numéricos, na seção 3 são apresentados os resultados das simulações numéricas e na seção 4 as conclusões.

\section{METODOLOGIA \\ 0 modelo atmosférico do CPTEC}

Neste trabalho os experimentos atmosféricos são realizados com o modelo atmosférico CPTEC/COLA descrito em detalhes em Cavalcanti et al. (2002). Este modelo é usado operacionalmente no CPTEC, tanto para previsão de tempo, quanto para previsão de clima. A versão utilizada tem 28 níveis na vertical e truncamento no número de onda sessenta e dois. As equações primitivas deste modelo incluem as equações da continuidade de massa para 0 ar seco e vapor d'água, a primeira lei da termodinâmica e as equações do movimento. Este modelo inclui uma formulação explícita da vegetação sobre a superfície da Terra e seus intercâmbios com a atmosfera e o solo através de uma versão simplificada do SIB (Simple Biosphere Model) Xue et al. (1991). A quantidade de nuvens convectivas é baseada na parametrização de convecção profunda proposta por Kuo (1974) e Anthes (1977). Detalhes deste modelo podem ser encontrados em Kinter III et al. (1997) e Cavalcanti et al. (2002).

\section{Simulação de Controle}

0 campo de TSM do NCEP (Reynolds \& Smith, 1994) a partir de novembro de 2000 até fevereiro de 2001 é usado como condição de contorno e as análises das 1200 UTC do NCEP dos dias 01 de novembro de 1995, 1996, 1998, 1999 e 2000 como condições iniciais de 5 integrações. 0 resultado aqui considerado é a média das integrações destas cinco condições iniciais para o período de 
dezembro de 2000 a fevereiro de 2001. Ou seja, o primeiro mês de integração é desconsiderado, desta forma evita-se a influência das condições iniciais na solução final das integrações. A média do conjunto é aqui denominada de integraçãa de controle (CTR). As cinco condições iniciais consideradas foram escolhidas de forma aleatória; optou-se por um conjunto de cinco membros levando em consideração a limitação do tempo de uso do supercomputador do CPTEC e também este é um número usado em diversos trabalhos que fazem experimentos de sensibilidade à TSM, como por exemplo em Ferranti et al. (1994).

Chaves (2005b) fez a avaliação desta integração CTR através de comparações com os dados de umidade específica, pressão ao nível do mar e vento da reanálise do NCEP/NCAR para o período de dezembro de 2000 a fevereiro de 2001. A habilidade dos modelos numéricos em simular o clima é essencial para que estes possam ser empregados em testes de hipóteses. A avaliação da integração CTR mostrou que este modelo simulou o clima observado para o período aqui considera, dando confiança ao seu uso.

0 campo de TSM (Fig. 1a) para o período de novembro de 2000 até fevereiro de 2001 é escolhido para ser usado como condição de contorno desta simulação CTR em função do Pacífico equatorial e maior parte das áreas oceânicas apresentarse com anomalias de TSM pouco intensas (Fig. 1b), coincidindo com a fase final do evento La-Niña de 2000/2001. Desta forma, 0 efeito das condições atmosféricas e oceânicas do Pacíico equatorial na Bacia do Atlântico e Índico é minimizado e o resultado das integrações nesta bacia é função principalmente da TSM local. Nas regiões subtropicais do Atlântico Sul e Norte e no Pacífico Noroeste são verificadas anomalias positivas de TSM acima de $1^{\circ} \mathrm{C}$.

\section{Experimentos com anomalias positivas e negativas de TSM sobre o Atlântico Norte}

Nos experimentos numéricos as anomalias negativas e positivas de TSM são impostas na área entre 0 Equador a $40^{\circ} \mathrm{N}$ e $70^{\circ} \mathrm{W}$ a $0^{\circ}$ com a finalidade de verificar a importância da TSM desta região na convecção de verão da América do Sul. Estes experimentos são denominados AFN e AQN, para os experimentos com anomalias negativas e positivas, respectivamente. Os campos de TSM impostos nesta bacia nos experimentos AFN $\mathrm{A}$ AQN são dados pelas Equações (1) e (2):

$$
\begin{gathered}
T S M_{A F N}= \\
T S M_{C T R}-\left[1+a b s\left(T S M_{C T R}-T S M_{C L I}\right]\right.
\end{gathered}
$$

$$
\begin{gathered}
T S M_{A Q N}= \\
T S M_{C T R}-\left[1+a b s\left(T S M_{C T R}-T S M_{C L I}\right]\right.
\end{gathered}
$$

nas quais os subscritos $C L I, C T R, A F N$ e $A Q N$ se referem aos campos de TSM climatológica e dos experimentos CTR, AFN e AQN, respectivamente. A climatologia aqui considerada refere-se à média da TSM entre 1981 e 2000 dos dados de TSM do NCEP (Reynolds \& Smith, 1994).

As anomalias de TSM com mesmo sinal são impostas sobre 0 Atlântico Norte na área mencionada no parágrafo acima, levandose em conta o fato do primeiro padrão da Análise de Componentes Principais das anomalias não normalizadas da TSM do Atlântico Sul apresentar o mesmo sinal em quase toda a área aqui considerada para o período de 1979 a 2001 (Chaves 2005a). Apesar do terceiro modo de variabilidades das anomalias de TSM do Atlântico Norte ser o principal modo acoplado com a convecção do setor norte da América do Sul (Chaves 2005a), optou-se pela estrutura do primeiro padrão de variabilidade das anomalias TSM como uma forma de intensificar a resposta da atmosfera à TSM.

Assim como na simulação CTR, nos experimentos AFN e AQN as análises das 1200 UTC do NCEP dos dias 01 de novembro de 1995, 1996, 1998, 1999 e 2000 são utilizadas como condições iniciais. O MCGA é integrado por 120 dias e os últimos 90 dias da média do conjunto são analisados, uma vez que a média do conjunto representa 0 sinal forçado pela condição de contorno da TSM (Ferranti et al., 1994). A sensibilidade do modelo às mudanças na intensidade da TSM é avaliada através da diferença entre a média da radiação de onda longa (ROL) da simulação CTR e do experimento considerado.

A significância estatística da diferença entre a média da ROL da simulação CTR e dos experimentos AFN e AQN é verificada usando 0 teste de duas caudas de $t$-Student's, como em Ferranti et al. (1994):

$$
t=\frac{X_{1}-X_{2}}{\sqrt{\left(S_{1}^{2}-S_{1}^{2}\right) / n}}
$$

na qual $X_{1}$ e $S_{1}$ são a média do conjunto e 0 desvio padrão da simulação controle e $X_{2}$ e $S_{2}$ são a média e 0 desvio padrão do experimento com a TSM anômala, respectivamente, e $n 0$ número de elementos do conjunto. Para dois conjuntos de cinco membros, com 8 graus de liberdade, 0 nível de significância de $95 \%$ para uma resposta estatisticamente significativa requer $|t|>1,99$.

\section{RESULTADOS}

Comparando a média da ROL da simulação CRT (Fig. 2a) com a do experimento AFN (Fig. 2b), nota-se que a desintensificação 
a

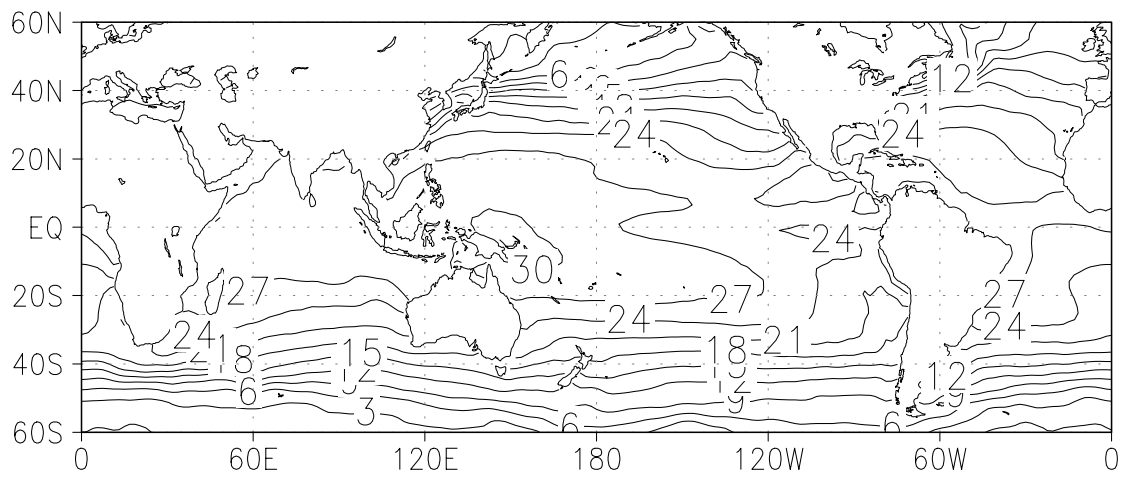

b

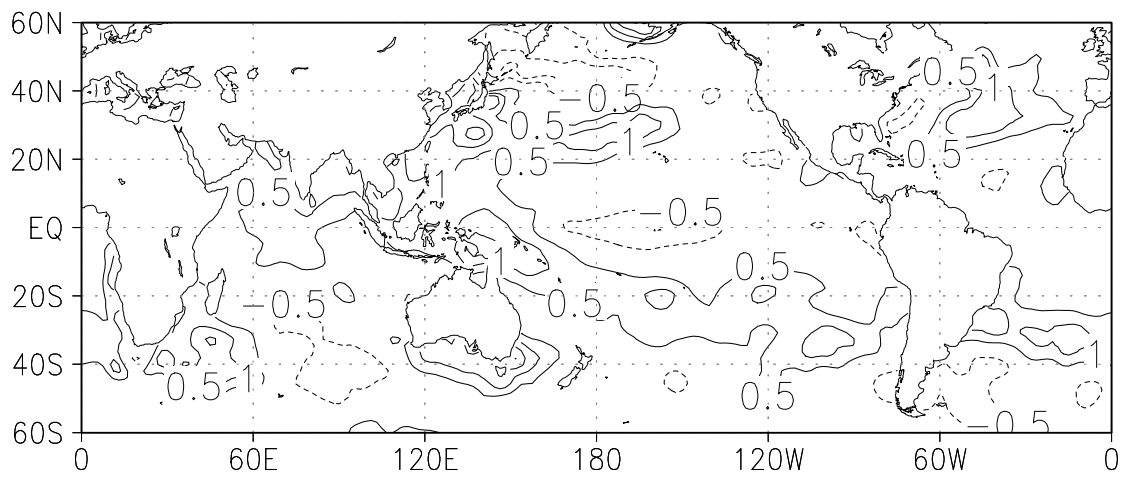

Figura 1 - Média da TSM observada (a) e da anomalia de TSM (b) $\left({ }^{\circ} \mathrm{C}\right)$ para 0 período de novembro de 2000 a fevereiro de 2001. Fonte: NCEP (Reynolds \& Smith, 1994).

da convecção na porção atlântica da ZCIT e norte da América do Sul (Fig. 3a) e a intensificação da convecção no norte do NEB e nas latitudes subtropicais da América do Sul (Fig. 3a) estão associada com o resfriamento da TSM do Atlântico Norte. No entanto, a diferença entre a simulação CTR e 0 experimento AFN tem significância estatística apenas sobre o norte da América do Sul e porção Atlântica da ZCIT.

No experimento AQN, a intensificação da convecção sobre a ZCIT e sobre a costa norte da América do Sul (Fig. 2c) está associada com as anomalias positivas de TSM sobre 0 Atlântico Norte. Estes resultados são estatisticamente significativos (Fig. 3b). Independente do sinal da anomalia imposta no campo de TSM utilizado no Atlântico Norte para forçar o modelo, na região da ZCAS a convecção apresenta-se menos intensa em relação à simulação CTR (Fig. 2), porém este resultado não apresenta significância estatística (Fig. 3).

No experimento AFN (Fig. 4b), a Alta Subtropical do Atlântico
Norte encontram-se mais intensa em relação à simulação CRT (Fig. 4a), e 0 padrão NAO encontra-se na sua fase positiva, com aumento da pressão sobre a Alta do Açores e diminuição sobre a Baixa da Iceland. No experimento AFN, associado com a intensificação da Alta dos Açores, os alísios de nordeste encontram-se mais intensos na costa norte da América do Sul, aumentando a divergência de umidade nesta região (Fig. 4b, 5a), sendo coerente com a desintensificação da convecção sob esta região (Fig. 3a). No lado leste dos Andes, a componente meridional do escoamento apresenta-se mais intensa em relação à simulação CTR. Este comportamento do escoamento a leste do Andes, provavelmente, contribui com a intensificação da convecção nas latitudes subtropicais da América do Sul quando 0 Atlântico Norte encontra-se mais frio (Fig. 3b). No experimento AQN (Fig. 4c) a Alta do Açores e os centros de alta pressão do Pacífico e Atlântico Sul apresentam-se menos intensos em relação aos experimentos CTR e AFN (Fig. 4a, b; 5b). 

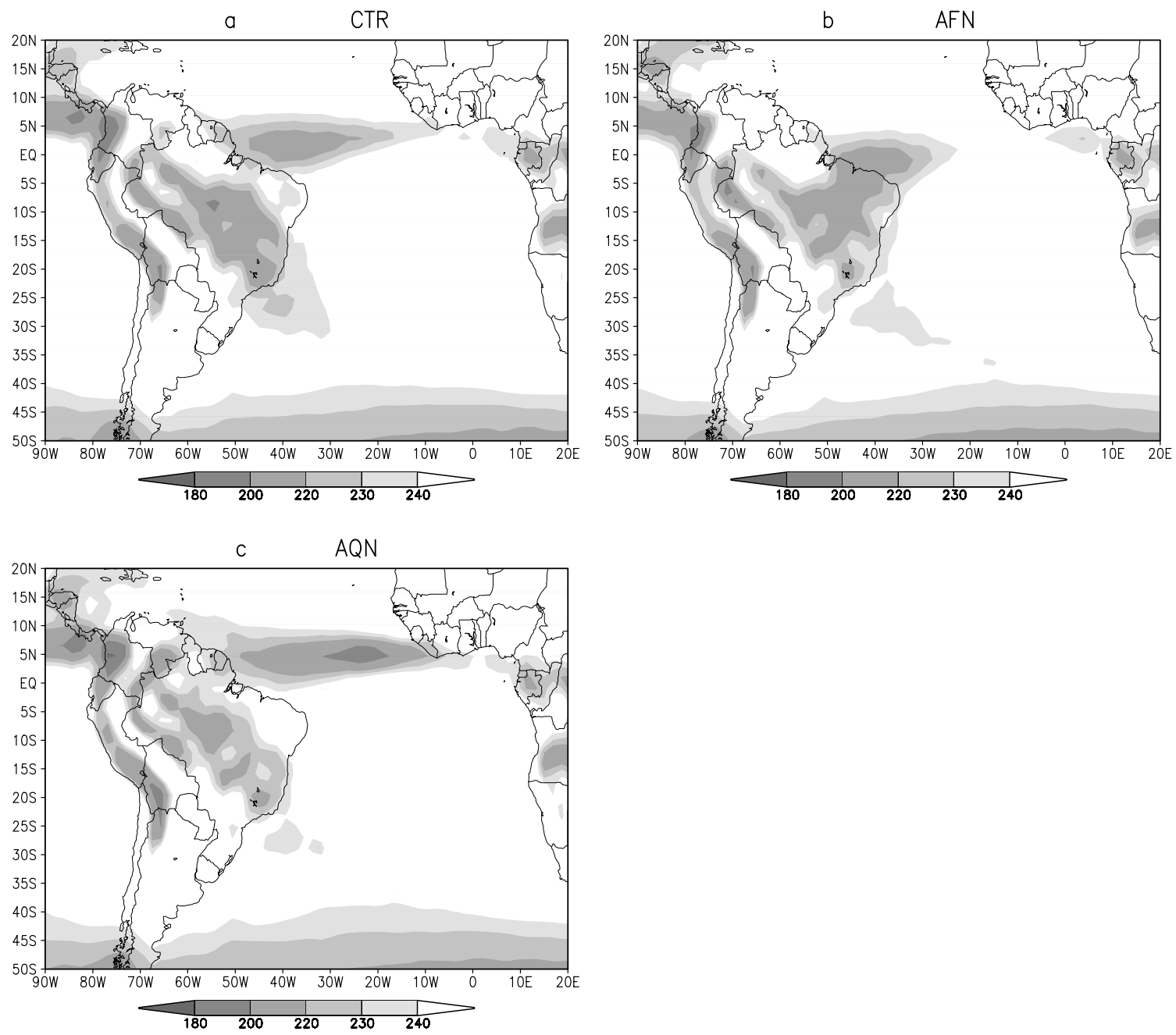

Figura 2 - Média de ROL (W/m²) para os experimentos CTR (a), AFN (b) e AQN (c).

Os resultados aqui apresentados são consistentes com o trabalho observacional de Wang \& Fu (2002), estes mostram que a intensificação da precipitação sobre o norte da América do Sul está associada com a desintensificação da componente meridional do vento a leste dos Andes. Enquanto a intensificação do escoamento a leste dos Andes está associada com 0 aumento da precipitação sobre a Bacia da Amazônia e a região subtropical da América do Sul.

\section{CONCLUSÕES}

Neste estudo a associação entre TSM do Oceano Atlântico Norte (Equador e $40^{\circ} \mathrm{N}$ e $70^{\circ} \mathrm{W}$ a $0^{\circ}$ ) e a convecção sobre a América do Sul é examinada através de simulações numéricas com o modelo atmosférico do CPTEC impondo-se anomalias positivas e negativas sobre esta área oceânica. Os resultados obtidos com as simulações numéricas mostram que a influência da TSM da região do Atlântico Norte aqui considerada sobre a convecção da América do Sul é significativa apenas no setor norte deste continente no período de DJF, corroborando os resultados obtidos no estudo observacional de Chaves (2005a) para DJF de 1979 a 2001. A convecção sobre este setor da América do Sul está associada com águas quentes sobre o Atlântico tropical Norte. Assim, observa-se também no modelo do CPTEC que a TSM sobre a área aqui considerada do Atlântico Norte tem pouca influência na previsibilidade da convecção sobre maior parte da América Sul nos meses de verão.

Para verificar a robustez dos resultados aqui apresentados serão necessários estudos de estudo de intercomparação com outros modelos e com outras resoluções para assegurar que es- 

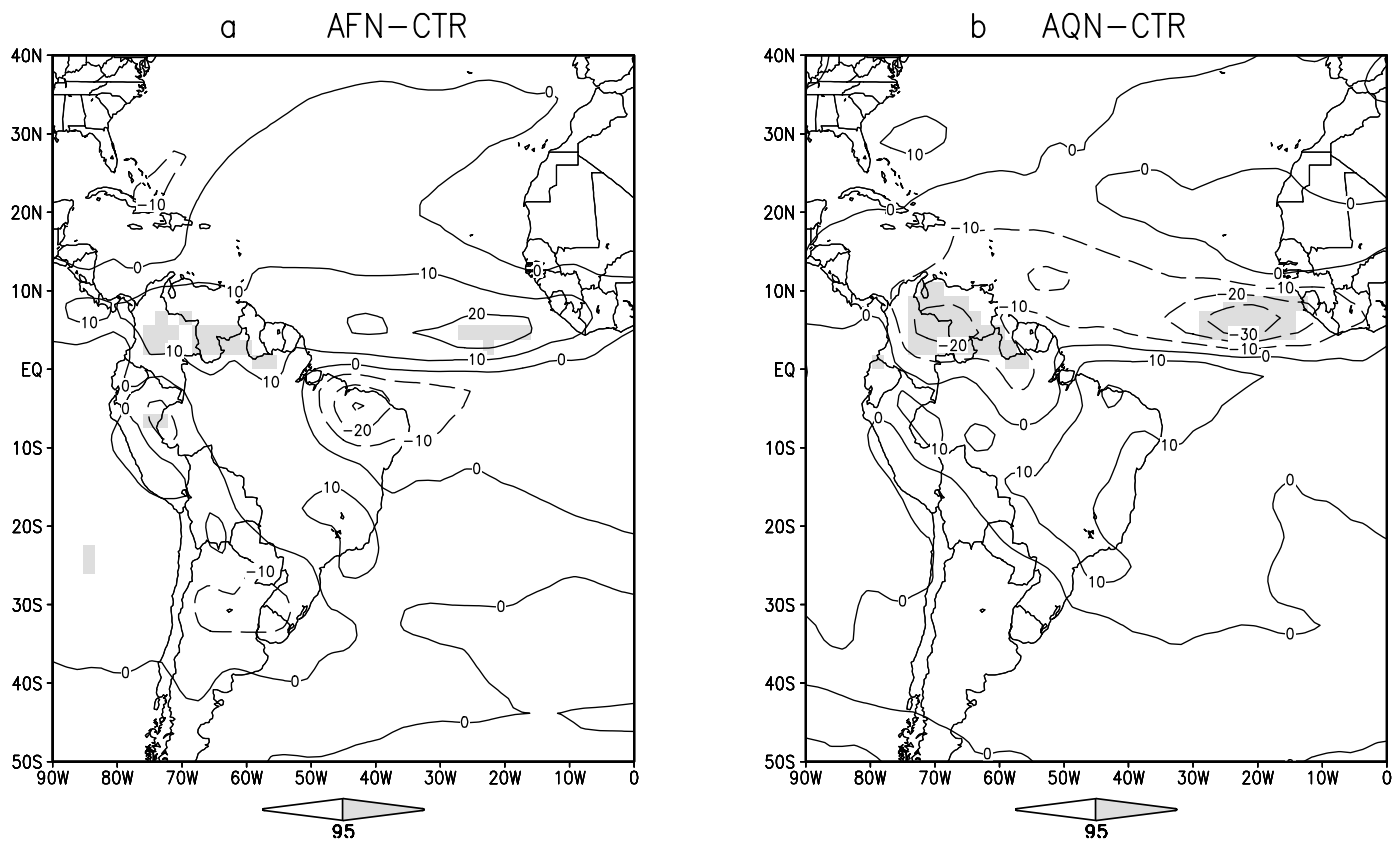

Figura 3 - Diferença entre ROL dos experimentos AFN e CTR (a) e AQN e CTR (b). Tom de cinza representa diferença com nível de significância acima de $95 \%$ segundo $0 t$-Student's.

tes resultados não são dependentes do modelo e da resolução; a realização dos mesmos experimentos com um número maior de membros no "ensemble"; verificação dos resultados obtidos com um modelo acoplado, ou ainda intercomparação com os dados de modelos acoplados de diversas fontes, a fim de verificar se os resultados aqui apresentados são verdadeiros no sistema acoplados.

\section{AGRADECIMENTOS}

A autora agradece ao Dr. Prakky Satyamurty pelas sugestões. Este trabalho é parte da Tese de Doutorado da autora defendida no INPE em agosto de 2003, financiada pelo CNPq.

\section{REFERÊNCIAS}

ANTHES RA. 1977. A Cumulus Parameterization Scheme Utilizing a OneDimensional Cloud Model. Mon. Wea. Rev., 105: 270-286.

BUCHMANN J, PAEGLE J, BUJA LE \& DICKINSON RE. 1990. The effect of tropical Atlantic heating anomalies upon GCM rain forecasts over the Americas. J. Clim., 3: 189-208.

CAVALCANTI IFA, MARENGO JA, SATYAMURTY P, NOBRE CA, TROSNIKOV I, BONATTI JP, MANZI AO, TARASOVA T, D'ALMEIDA C, SAMPAIO G, PEZZI LP, CASTRO CC, SANCHES M \& CAMARGO H. 2002. Global climatological features in a simulation using CPTEC/COLA AGCM. J. Clim., 15: 2965-2988.
CHAVES RR. 2005a. Padrões da temperatura da superfície do Oceano Atlântico Norte e a convecção de verão sobre a América do Sul - análise observacional. Revista Brasileira de Geofííca, 23: 27-38.

CHAVES RR. 2005b. Resposta de um Modelo de Circulação Geral do Oceano ao Campo de Stress de Vento Produzido por um Modelo de Circulação Geral da Atmosfera e Reanálises. Revista Brasileira de Meteorologia, 20: 437-450.

DOMMENGET D \& LATIF M. 2000. Interannual to decadal variability in the tropical Atlantic. J. Clim., 13: 777-792.

FERRANTI L, MOLTENI F \& PALMER TN. 1994. Impact of localized tropical and extratropical SST anomalies in ensembles of seasonal GCM integrations. Quart. J. Roy. Meteor. Soc., 120: 1613-1645.

FU R, DICKINSON RE, CHEN M \& WANG H. 2001. Tropical sea surface temperatures influence the seasonal distribution of precipitation in the Equatorial Amazon? J. Clim., 14: 4003-4026.

HASTENRATH S \& HELLER L. 1977. Dynamics of climatic hazards in Northeast Brazil. Quart. J. Roy. Meteor. Soc., 103: 77-92.

HOUGHTON RW \& TOURRE YM. 1992. Characteristics of low-frequency sea surface temperature fluctuations in the tropical Atlantic. J. Clim., 5: 765-771.

KINTER III JL, DEWITT D, DIRMEYER P, FENNESSY M, KIRTMAN B, MARX L, SCHNEIDER E, SHUKLA J \& STRAUS D. 1997. The COLA Atmosphere-Biosphere General Circulation Model Volume: 1 Formulation. COLA 51, $46 \mathrm{pp}$. 

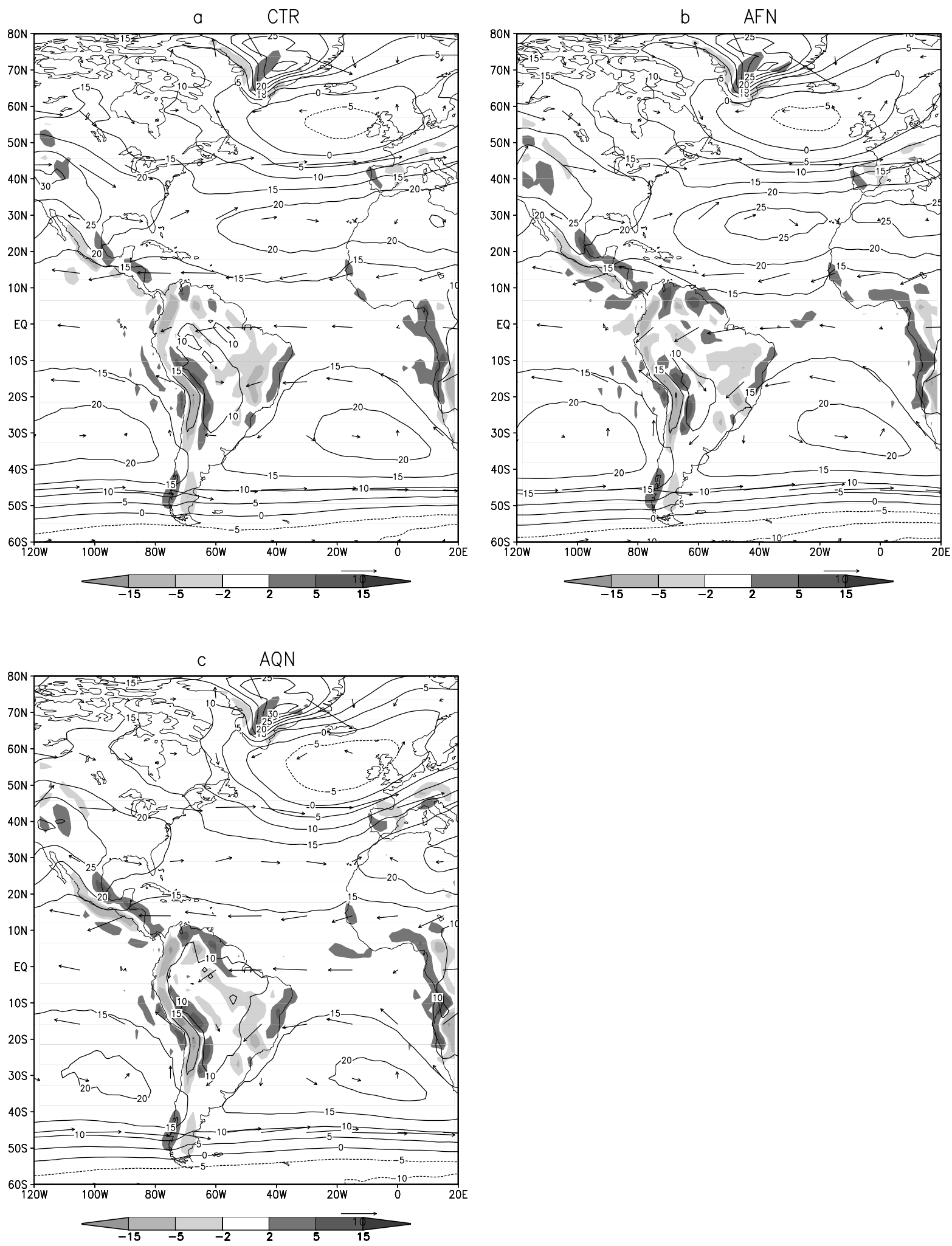

Figura 4 - Campo de vento (m/s) e divergência de umidade específica (tons de cinza; g/Kg/dia) integrado na vertical entre 1000 e $500 \mathrm{hPa}$ e campo de pressão ao nível do mar (isolinhas; $+1000 \mathrm{hPa}$ ) para os experimentos CTR (a), AFN (b) e AQN (c). 

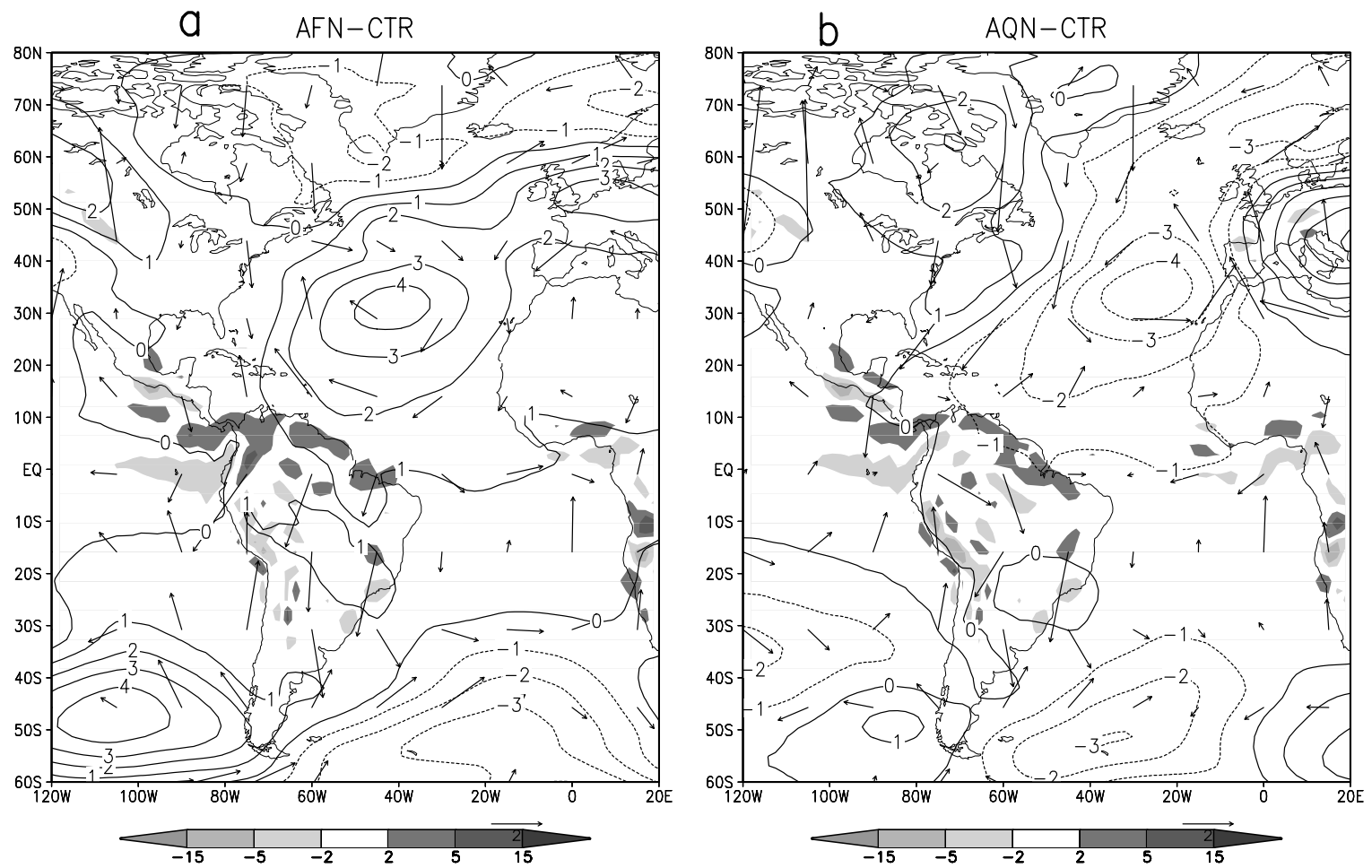

Figura 5 - Diferença entre o campo de vento (m/s) e divergência de umidade específica (tons de cinza; $\mathrm{g} / \mathrm{Kg} / \mathrm{dia}$ ) integrado na vertical entre 1000 e $500 \mathrm{hPa}$ e campo de pressão ao nível do mar (isolinhas; -1000 hPa) para os experimentos AFN-CTR (a) e AQN-CTR (b).

KUO KL. 1974. Further studies of the parameterization of the influence of cumulus convection on large scale flow. J. Atmos. Scie., 31: 1232-1240.

LIEBMANN B \& MARENGO JA. 2001. Interannual variability of the rainy season and rainfall in the Brazilian Amazon Basin. J Clim., 14: 43084318.

MOURA AD \& SHUKLA J. 1981. On the dynamics of droughts in Northeast Brazil: observations, theory and numerical experiments with a general circulation model. J. Atmos. Sci., 38: 2653-2675.

NOBRE P \& SHUKLAJ. 1996. Variations of sea surface temperature, wind stress, and rainfall over the tropical Atlantic and South America. J. Clim., 9: 2464-2479.

REYNOLDS RW \& SMITH TM. 1994. Improved global sea surface temperature analyses using optimum interpolation. J. Clim., 7: 929-948.

XUE Y, SELLERS PJ, KINTER III JL \& SHUKLA J. 1991. A simplified biosphere model for global climate studies. J. Clim., 4: 345-364.

WANG F \& FU R. 2002. Cross-equatorial flow and seasonal cycle of precipitation over South America. J. Clim., 15: 1591-1608.

\section{NOTA SOBRE $O$ AUTOR}

Rosane Rodrigues Chaves é meteorologista pelo Instituto de Astronomia, Geofísica e Ciências Atmostéricas (IAG) da USP, com Mestrado e Doutorado em Meteorologia pelo INPE. Professora Associada da Universidade Estadual do Norte Fluminense Darcy Ribeiro - UENF no Laboratório de Meteorologia - LAMET e Laboratório de Engenharia e Exploração de Petróleo - LENEP. 